\title{
ON STARK BROADENING OF HEAVY ELEMENT LINES IN A-TYPE STAR SPECTRA: Bi II LINES
}

\author{
M. S. DIMITRIJEVIĆ and L. Č. POPOVIĆ \\ Astronomical Observatory, Volgina 7, 11050 Beograd, Serbia, Yugoslavia
}

\begin{abstract}
We report here results of $\mathrm{Bi}$ II spectral lines Stark broadening research within the modified semiempirical approach. The strong absorption $\mathrm{Bi}$ II lines observed in $\mathrm{Hg}-\mathrm{Mn}$ star atmospheres have been investigated as well as the influence of the departure from LS - coupling and the correct knowledge of ionization potential. The obtained results have been compared with other estimations and with experimental results.
\end{abstract}

\section{INTRODUCTION}

Stark broadening data are of the great importance for astrophysical and laboratory plasma spectroscopy. For evaluation and modelling of stellar atmospheric physical properties and abundace determinations, Stark broadening data for a large number of transitions in many atoms are needed.

Seven strong absorption lines of ionized bismuth have been found in the $\mathrm{Hg}-\mathrm{Mn}$ star HR 7775 in high - resolution spectra obtained with IUE (Jacobs and Dworetsky, 1982). Performed analysis shows existence of the overabundance of $\mathrm{Bi}$ of $10^{6}$ while Jacobs and Dworetsky (1982) have not detected Bi II in the spectra of several other $\mathrm{Hg}-\mathrm{Mn}$ stars. Since the plasma conditions in HR 7775 star atmosphere are $T_{\text {eff }}=11000 \mathrm{~K}$, $\log g=4.0$ (Jacobs and Dworetsky, 1982), it is of interest to provide the corresponding Stark broadening parameters which might be of significance for abundance investigation, determination of astrophysical $g f$ values and other stellar plasma research. Besides of an astrophysical importance, Stark broadening of Bi II lines is interesting and for labaratory plasma research and was investigated experimentally by Miller and Bengston (1980) and Puric et al., (1985). Moreover the case of $\mathrm{Bi}$ II lines is interesting from the theoretical point of view since this is an example of depature from LS - coupling which gives the opportuity to study influence of such effect on Stark broadening parametars.

\section{THEORY}

According to modified semiempirical approach, electron impact width (HWHM) of an ion line is given by the experssion (Dimitrijević and Konjević, 1980):

$$
\begin{gathered}
w=N \frac{2 h^{2}}{3 m^{2}}\left(\frac{2 m}{3 \pi k T}\right)^{1 / 2} \cdot\left\{\vec{R}_{l_{i}, l_{i+1}}^{2} \tilde{g}\left(x_{l_{i}, l_{i+1}}\right)+\vec{R}_{l_{i}, l_{i-1}}^{2} \tilde{g}\left(x_{l_{i}, l_{i-1}}\right)+\vec{R}_{l_{f}, l_{f+1}}^{2} \tilde{g}\left(x_{l_{f}, l_{f+1}}\right)+\right. \\
\left.\vec{R}_{l_{j}, l_{f-1}}^{2} \tilde{g}\left(x_{l_{f}, l_{f-1}}\right)+\sum_{i^{\prime}}\left(\vec{R}_{i^{\prime}}^{2}\right)_{\Delta n \neq 0} g\left(x_{n_{i}}\right)+\sum_{f^{\prime}}\left(\vec{R}_{f f^{\prime}}^{2}\right)_{\Delta n \neq 0} g\left(x_{n_{f}}\right)\right\}
\end{gathered}
$$




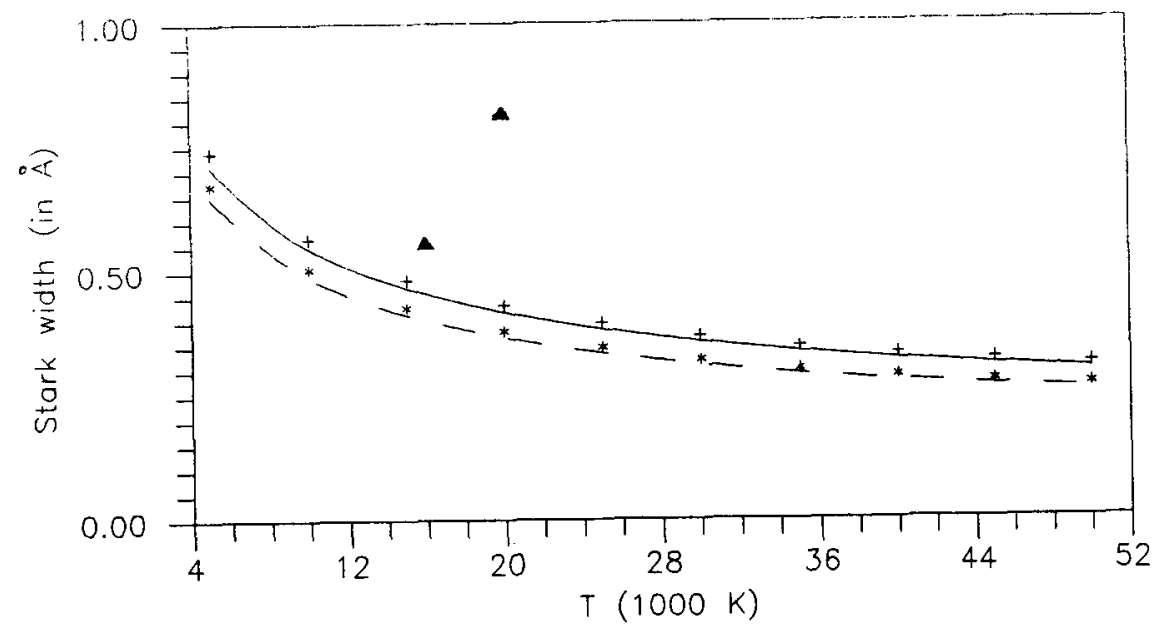

Fig. 1. Stark width (HWHM) for Bi II $5209 \AA\left(7 \mathrm{~s}^{3} \mathrm{P}_{1}^{0}-7 \mathrm{p}^{3} \mathrm{D}_{2}\right)$ spectral line as a function of temperature $(T)$, at electron density $N=10^{17} \mathrm{~cm}^{-3}$.

The used notation is: (-) - present result with $E_{\text {ion }}=127000 \mathrm{~cm}^{-1}$; (- - ) - present results with $E_{i o n}=134600 \mathrm{~cm}^{-1} ;(+++)-E_{i o n}=127000 \mathrm{~cm}^{-1}$ and pure LS coupling assumption; $(* * *)-E_{\text {ion }}=134600 \mathrm{~cm}^{-1}$ and pure LS coupling assumption; $\oplus$ - estimate of Lakićević (1983); $\boldsymbol{\Lambda}$ - experimental data of Purić et al. (1985).

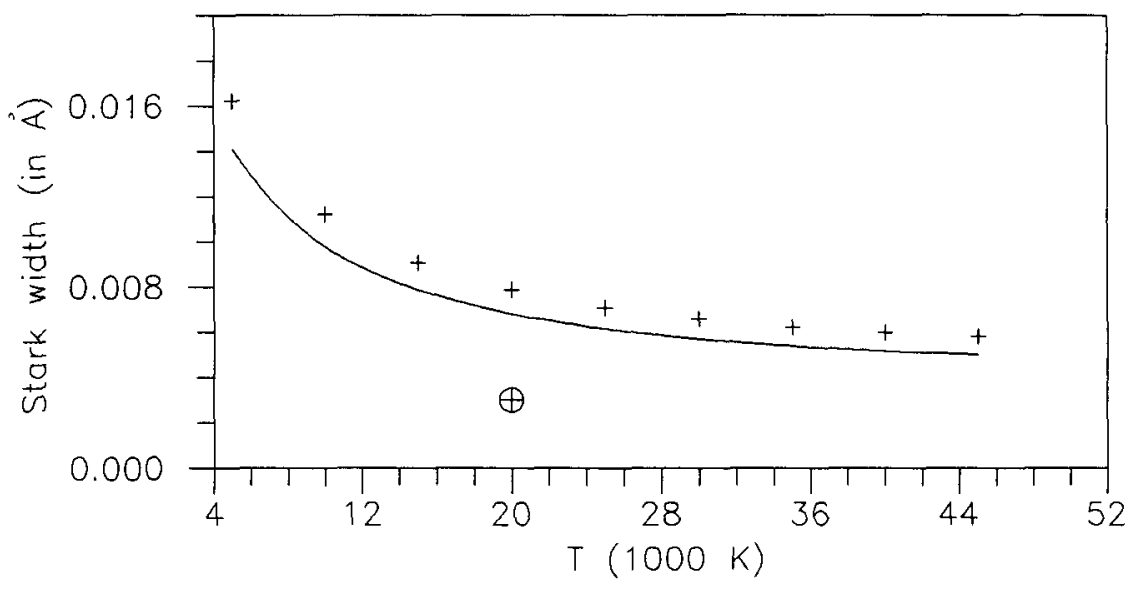

Fig. 2. Same as in Fig. 1. but for Bi II $1436.83 \AA$ spectral line $\left(6 \mathrm{p}^{2}{ }^{3} \mathrm{P}_{0}-7 \mathrm{~s}{ }^{3} \mathrm{P}_{1}^{0}\right)$. 


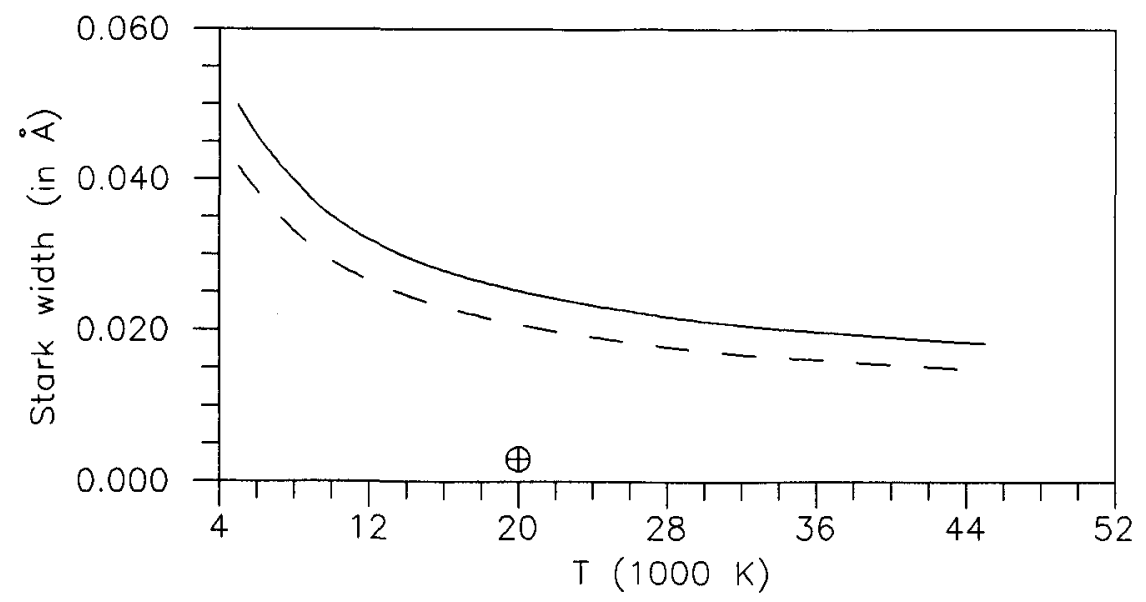

Fig. 3. Same as in Fig. 1. but for Bi II $1325.46 \stackrel{A}{A}$ spectral line $\left(6 \mathrm{p}^{2}{ }^{3} \mathrm{P}_{1}-7 \mathrm{~s}^{3} \mathrm{P}_{2}\right)$.

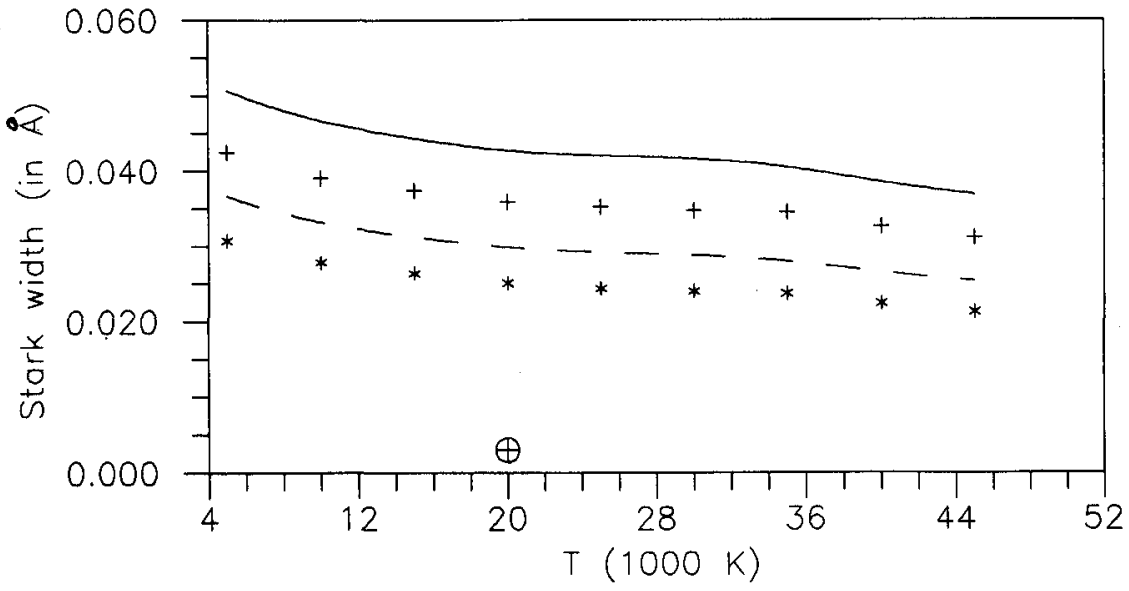

Fig. 4. Same as in Fig. 1. but for Bi II $1372.61 \AA$ spectral line $\left(6 \mathrm{p}^{2}{ }^{3} \mathrm{P}_{2}-7 \mathrm{~s}{ }^{1} \mathrm{P}_{1}^{0}\right)$. where $\vec{R}_{j j^{\prime}}^{2}(j=i, f)$ is the square of the coordinate operator matrix element

$$
\begin{gathered}
\vec{R}_{l, l^{\prime}}^{2} \approx\left(\frac{3 n^{*}}{2 Z}\right)^{2} \frac{\max \left(l, l^{\prime}\right)}{2 l+1}\left[n^{* 2}-\max ^{2}\left(l, l^{\prime}\right)\right] \varphi^{2} \\
\sum_{j^{\prime}}\left(\vec{R}_{j j^{\prime}}^{2}\right)_{\Delta n \neq 0} \approx\left(\frac{3 n^{*}}{2 Z}\right)^{2} \frac{1}{9}\left(n_{j}^{* 2}+3 l_{j}^{2}+3 l_{j}+11\right) .
\end{gathered}
$$

In Eqs. 1-3 with $i, f, i^{\prime}, f^{\prime}$ are denoted initial and final level respectively and their corresponding perturbing levels, $l_{j}$ is the angular momentum quatum number, $\varphi$ is the Bates-Damgaard factor (Bates and Damgaard, 1949; Oertel and Shomo, 1968), $g$ 
factors, $x_{j, j^{\prime}}=3 k T / 2\left|\Delta E_{j, j^{\prime}}\right|\left(\Delta E_{j j^{\prime}}=E_{j^{\prime}}-E_{j}\right), T$ is electron temperature, $x_{n_{j}}=$ $3 k T n_{j}^{* 3} / 4 Z^{2} E_{H}\left(E_{H}\right.$ is hydrogen ionization energy, $Z$ is residual ionic charge, $Z=1$ for neutrals, 2 for single charged ions, etc.), $n_{j}^{*}=\left[E_{H} Z^{2} /\left(E_{i o n}-E_{j}\right)\right]^{1 / 2}$ is effective principal quantum number ( $E_{i o n}$ is appropriate spectral series limit), and $N$ is electron density.

For $6 \mathrm{p}^{2}-6 \mathrm{p} 7 \mathrm{~s}$ transitions we derived the needed Bates and Damgaard factors $\varphi$ from oscillator strengths calculated by Gruzdev (1968). Moreover, Gruzdev demonstrated that departure form LS coupling of $6 \mathrm{p}^{2}$ and $6 \mathrm{p} 7 \mathrm{~s}$ terms may be taken into account by representing the corresponding term as, a mixture of singlet and triplet states, and provided corresponding partition coefficient. For example $6 \mathrm{p}^{2}{ }^{3} \mathrm{P}_{2}$ may be represented as a mixture of $K_{1} 6 \mathrm{p}^{2}{ }^{3} \mathrm{P}_{2}$ and $K_{2} 6 \mathrm{p}^{2}{ }^{1} \mathrm{D}_{2}$, where $K_{1}+K_{2}=1$. We calculated the corresponding squares of the coordinate operator matrix element in the form

$$
\vec{R}_{j j^{\prime}}^{2}=K_{\alpha} \cdot \vec{R}_{j j^{\prime}}^{2}+K_{\beta} \cdot \vec{R}_{\alpha \alpha^{\prime}}^{2}
$$

where with $\alpha, \alpha^{\prime}$ are denoted the part with other multiplicity and the corresponding perturbing levels.

\section{RESULTS AND DISCUSSION}

Results for half width at halfmaximum for four Bi II lines are presented in Figs. 1 4 and compared with existing experimental data (Puric et al., 1985) as well as with the simple estimation of Lakićević (1983) based on regularities and systematic trends. In the case of $\mathrm{Bi}$ II spectrum exist an additional uncetrainty. Namely the ionization potential $\left(E_{i o n}\right)$ of $134600 \mathrm{~cm}^{-1}$ given in Moore's tables (1971) is too high according to Gruzdev's analysis (1968); who suggest a lower value of $127000 \mathrm{~cm}^{-1}$. In Figs. $1-$ 4 with solid line are denoted results obtained by $E_{\text {ion }}=127000 \mathrm{~cm}^{-1}$ and with dashed line result obtained with $E_{i o n}=134600 \mathrm{~cm}^{-1}$. With crosses and asterisks aree denoted calculation with two $E_{\text {ion }}$ respectively, when pure IS coupling is assumed. We can see that the influence of both factors depends on transition. Taking into account that the accuracy of presented estimates is low due to complexity of the $\mathrm{Bi}$ II spectrum, these differences might give an impression on the accuracy of Bi II Stark width data.

\section{REFERENCES}

Bates, D. R. and Damgaard A., 1949, Phyl. Trans. Roy. Soc. London A242, 101.

Gruzdev, P. F., 1968, Opt. Spect., 25, 3.

Griem, H. R., 1968, Phys. Rev., 165, 258.

Dimitrijević, M. S. and Konjević, N., 1980, JQSRT., 24, 451.

Jacobs, J. M. and Dworetsky, M. M., 1982, Nature, 299, 535.

Lakićević, S. I., 1983, Astron. Astrphys., 127, 37.

Miler, M. H. and Bengtson, R. D., 1980, JQSRT., 23, 411.

Moore, C. E.: 1971, Atomic Energy Levels III, NSRDS - NBS35, US Govt. Printing Office, Washington DC.

Oertel, G. K. and Shomo, L. P., 1968, Astrophys. J. Suppl. Series, 16, 175.

Purić, J., Ćuk, M. and Lakićević, I. S., 1985, Phys. Rev., A 32, 1106. 